\title{
Operational Calculus in Several Variables
}

\author{
By Tetsuo WATANABE \\ The National Defense Academy \\ (Communicated by Kiyosi ITô, M. J. A., May 12, 1995)
}

1. Introduction. The Theory of Mikusinski's operational calculus is based on the Theorem of Titchmarsh which guarantees that the ring of the complexed-valued continuous functions with convolution as product is an integral domain. He treated a partial differential equation of two variables with constant coefficients as an ordinary differential equation with coefficients in the convolution ring of one variable. But in order to treat equations more symmetrically with respect to several variables, we consider convolution product of functions of several variables. Then, if the differential operator of a partial differential equation with constant coefficients can be factored into linear operators, we are able to treat them just as in the case of one variable. To develope the theory in several variable case, natural definition of convolution of two functions $f\left(t_{1}, \cdots, t_{m}\right)$ and $g\left(t_{1}, \cdots, t_{m}\right)$ is

$$
\begin{aligned}
& (f * g)\left(t_{1}, \cdots, t_{m}\right)=\int_{0}^{t_{1}} \cdots \int_{0}^{t_{m}} \\
& \quad f\left(t_{1}-u_{1}, \cdots, t_{m}-u_{m}\right) g\left(u_{1}, \cdots, u_{m}\right) d u_{1} \cdots d u_{m},
\end{aligned}
$$
since we can easily verify that the ring $C$ of $m$ variables on $[0, \infty)^{m}$ is a commutative ring with this product. It is known ([1]) that this ring is an integral domain. We define partial integration operators and linear partial differential operators based on this theorem and as applications we consider linear differential equations with constant coefficients.

\section{The convolution quotients.}

Theorem 1 (Generalized Titchmarsh's theorem). Let $A$ be the convolution ring of continuous functions on $R^{m}$ which have support in [0, $\infty)^{m}$. Then $A$ has no divisors of zero.

The proof is in [1] Chapter VI.

To simplify notation we shall consider two variable case. Therefore we denote by $C$ the convolution ring of complex-valued continuous functions $z(x, y)$ with support in $[0, \infty)^{2}$ which are continuously differentiable on $(0, \infty)^{2}$ with continuously differentiable $z(x, 0)$ and $z(0, y)$. We assume that all functions in this paper are in $C$ and $\alpha>0$ and $c$ a complex number. For $f(x, y)$, $g(x, y)$ in $C$, let $f * g$ be the convolution of $f$ and $g$ with respect to $x, y$, that is,

$$
\begin{array}{r}
(f * g)(x, y) \\
\quad=\int_{0}^{x} d u \int_{0}^{v} f(x-u, y-v) g(u, v) d v .
\end{array}
$$

Then we can easily verify

$$
\begin{aligned}
f * g & =g * f, \\
f *(g * h) & =(f * g) * h .
\end{aligned}
$$

With the usual sum $f(t)+g(t)$, it is clear that

$$
h *(f+g)=h * f+h * g .
$$

We often omit ' $*$ ' if it is clear from the context. To stress that we consider a function $f(x, y)$ as an element of the $\operatorname{ring} C$, we denote it by $\{f(x$, $y)\}$. By the generalized Titchmarsh's theorem, the ring $C$ is without zero-divisor. Let $Q$ be the field of total fractions of $C$ and we shall call each element of $Q$ an inner operator and any other operator operating on $C$ or $\boldsymbol{Q}$ an outer operator. Functions in $C$ may not necessarily be continuous on $x$ and $y$ axes, but we have

Proposition 2. The ring $C$ has no divisors of zero.

Proof. Let $f, g$ be in $C$ and $f * g=0$. Then $1 * f$ and $1 * g$ are in $A$. Since $(1 * f) *(1 * g)=$ 0 , either $1 * f=0$ or $1 * g=0$. Differentiating 1 $* f=0$ or $1 * g=0$ by $x$ and $y$, we have either $f(x, y)=0$ or $g(x, y)=0$ for $(x, y)$ in $(0, \infty)^{2}$. Since $f, g$ are continuous on $[0, \infty)^{2}$ and with support in $[0, \infty)^{2}$, we have $f(x, y)=0$ or $g(x$, $y)=0$ identically.

Definition. We define the unit operator $I$ by $I=\frac{\{1\}}{\{1\}}$.

We shall denote by $h$ the operator defined by the function $\{1\}$ :

$$
h=\{1\} \text {. }
$$

Then, for any natural number $n$, we have, by induction on $n$,

$$
h^{n}=\left\{\frac{x^{n-1} y^{n-1}}{(n-1) !(n-1) !}\right\} .
$$


For any complex number $\alpha$, the inner operator $[\alpha]=\{\alpha\} / h$ is called a scalar operator.

As in one variable case, we can easily verify:

$$
\begin{gathered}
{[\alpha]+[\beta]=[\alpha+\beta],} \\
{[\alpha] *[\beta]=[\alpha \beta],} \\
{[\alpha] *\{f(x, y)\}=\{\alpha f(x, y)\} .}
\end{gathered}
$$

We define the inner operator of "partial integration" $h_{k}$ by

$$
h_{x}=\{x\} / h \text { and } h_{y}=\{y\} / h \text {. }
$$

Therefore we identify $[\alpha]$ with $\alpha$, especially when it is a coefficient of a polynomial of operators.

Proposition 3. $h_{x}\{f(x, y)\}=\left\{\int_{0}^{x} f(u, y) d u\right\}$, $h_{y}\{f(x, y)\}=\left\{\int_{0}^{y} f(x, v) d v\right\}$.

We shall verify the first formula. By Proposition 2 , it is sufficient to show that

$$
\{x\}\{f(x, y)\}=\{1\}\left\{\int_{0}^{x} f(u, y) d u\right\} .
$$

We can verify the equality by expressing both sides according to the definition of involution product and then differentiating both sides by $x$ and comparing them, since both sides are zero if $x=0$.

Proposition 4. $h_{x} h_{y}=h$.

By induction, we have

\section{Proposition 5.}

$$
h_{x}^{j} h_{y}^{k}=\frac{1}{h}\left\{\frac{x^{j}}{j !} \frac{y^{k}}{k !}\right\} .
$$

Definition. Let $r_{x}$ be an operator on $C$ defined by $r_{x}\{f(x, y)\}=\{f(0, y)\}$.

$r_{y}$ is defined similarly.

Let $s_{x}=\frac{1}{h_{x}}, s_{y}=\frac{1}{h_{y}}, d_{x}=s_{x}-s_{x} r_{x}$ and $d_{y}$ $=s_{y}-s_{y} r_{y}$.

Proposition 6. Let $f$ be in $C$. Then

$$
\begin{aligned}
& \frac{\partial}{\partial x}\{f(x, y)\}=d_{x}\{f(x, y)\} \\
& \frac{\partial}{\partial y}\{f(x, y)\}=d_{y}\{f(x, y)\} .
\end{aligned}
$$

Proof. Since $h_{x}\left\{f_{x}(x, y)\right\}=\{f(x, y)-$ $f(0, y)\}$, we have the result by multiplying $s_{x}$ to both sides from the left.

From Proposition 2 and Proposition 6, we have

Corollary. Let $\alpha, \beta, \gamma$ be complex numbers. Then $z(x, y)$ in $C$ satisfying

$$
\alpha \frac{\partial z(x, y)}{\partial x}+\beta \frac{\partial z(x, y)}{\partial y}+\gamma z(x, y)=f(x, y)
$$

is determined uniquely by the values on $x=0$, $y \geq 0$ and $y=0, x \geq 0$.

From definitions, we can easily see:

Lemma 7.

$$
\begin{aligned}
& r_{x} r_{y}=r_{y} r_{x}, \\
& r_{x} h_{y}=h_{y} r_{x}, \\
& s_{y} r_{x}=r_{x} s_{y} .
\end{aligned}
$$

Lemma 8. Let $z(x, y)$ be in $C$ satisfying

$\left(\frac{\partial}{\partial x}-\frac{\partial}{\partial y}\right) z(x, y)=0 \quad(x>0, y>0)$.

Then we have $z(x, y)=z(0, x+y)=$ $z(x+y, 0)$.

Proof. Since both $z(x, y)+z(y, x)$ and $z(x$ $+y, 0)+z(0, x+y)$ are solutions of the differential equation and have the same values on both axes, we have $z(x, y)+z(y, x)=z(x+$ $y, 0)+z(0, x+y)$ by the uniqueness of the solution. Similarly we have $z(x, y) z(y, x)=$ $z(0, x+y) z(x+y, 0)$. Therefore either $z(x, y)$ $=z(0, x+y)$ or $z(x, y)=z(x+y, 0)$. Since $z(x, y)$ is continuous on the line $x+y=a$ and can only take the two values $z(0, a)$ and $z(a, 0)$, we know that $z(x, y)=z(0, a)=z(a, 0)$.

Since a function in $C$ remains in $C$ and its boundary condition on $y$-axis corresponds to the condition on $Y$-axis if we change the variables $X$ $=\alpha x, Y=y(\alpha>0)$ or multiply it by the function of the form $e^{c x}$, we have by Lemma 8:

Proposition 9. Let $z(x, y)$ be in $C$ satisfying $\left(\frac{\partial}{\partial x}-\alpha \frac{\partial}{\partial y}+c\right)\{z(x, y)\}=0 \quad(x>0, y>0)$. Then we have $z(x, y)=e^{-c x} z(0, \alpha x+y)=$ $e^{-c x} z(\alpha x+y, 0)$.

In particular, if $z(0, y)=0$ for all $y>0$, then $z(x, y)=0$ identically.

Proposition 10. Let $f(x, y)$ be in $C$. Then $z(x, y)=\int_{0}^{x} e^{c(u-x)} f(u, y+\alpha x-\alpha u) d u$ is the unique solution of $\left(s_{x}-\alpha d_{y}+c\right)\{z(x, y)\}=$ $\{f(x, y)\}$

with boundary condition $z(0, y)=0(y>0)$.

Proof. $z(x, y)$ is a solution by direct calculation and is unique by Proposition 9.

Definition. By Proposition 10, we can define

$$
\begin{gathered}
\frac{1}{s_{x}-\alpha d_{y}+c}\{f(x, y)\} \\
=\int_{0}^{x} e^{c(u-x)} f(u, y+\alpha x-\alpha u) d u .
\end{gathered}
$$

Lemma 11. Let $z(x, y)$ be a continuous func- 
tion on $[0, \infty)^{2}$, satisfying

$$
\left(d_{x}+\alpha d_{y}+c\right)\{z(x, y)\}=0
$$

and $z(0, y)=0$ for all $y \geq 0$. Then we have $z(x, y)$ $=0$ if $0 \leq \alpha x \leq y$.

Proof. By multiplying $z(x, y)$ by $e^{c x}$ and by the change of variables $X=\alpha x, Y=y$, we reduce the equation to the case of $\alpha=1, c=0$. We want to show that, if $\left(d_{x}+d_{y}\right) z(x, y)=0(x$ $<y)$ with $z(0, y)=0$ for all $y>0$, then $z(x, y)$ $=0$ on the domain $x<y$. By the change of variables $x=X, y=X+Y$, the domain $y>x \geq 0$ corresponds to $X \geq 0, Y \geq 0$. Let $w(X, Y)$ $=z(X, X+Y)$ and we shall show that $w(X, Y)$ $=0$ identically on the first quadrant. Since $d_{X} w=\frac{\partial w}{\partial X}=\frac{\partial z}{\partial x}+\frac{\partial z}{\partial y}=0$, we have $d_{X} w(X, Y)$ $=s_{X}(w(X, Y)-w(0, Y))=0$. Hence $w(X, Y)$ $=w(0, Y)=z(0, Y)=0$.

Proposition 12.

$$
\frac{1}{\left(s_{x}-\alpha\right)\left(s_{y}-\beta\right)}=\left\{e^{\alpha x+\beta y}\right\} .
$$

More generally we have

$$
\frac{1}{\left(s_{1}-\alpha_{1}\right) \cdots\left(s_{m}-\alpha_{m}\right)}=\left\{e^{\alpha_{1} x_{1}+\cdots+\alpha_{m} x_{m}}\right\},
$$

where $s_{j}=\frac{\left\{x_{j}\right\}}{\{1\}},(1 \leq j \leq m)$.

Proof. Let $|t|<1$ and $|s|<1$. Then we have

$$
\frac{1}{(1-t)(1-s)}=\sum_{j=0}^{\infty} \sum_{k=0}^{\infty} t^{j} s^{k}
$$

Since this series converges in the neighborhood of $(0,0)$, we can substitute $\alpha h_{x}, \beta h_{y}$ for $t, s$. Since

$$
\begin{aligned}
& \qquad\left(\alpha h_{x}\right)^{j}\left(\beta h_{y}\right)^{k}=\frac{1}{h}\left\{\frac{(\alpha x)^{j}}{j !} \frac{(\beta y)^{k}}{k !}\right\} \\
& \text { we have } \frac{1}{\left(1-\alpha h_{x}\right)\left(1-\beta h_{y}\right)}=\frac{1}{h}\left\{e^{\alpha x+\beta y}\right\}
\end{aligned}
$$

Multiplying $h=h_{x} h_{y}$ to both sides, we have $\frac{1}{\left(s_{x}-\alpha\right)\left(s_{y}-\beta\right)}=\left\{e^{\alpha x+\beta y}\right\}$.

Definition. We define algebraic partial derivations $D_{k}$ with respect to $s_{k}(1 \leq k \leq m)$ by $D_{k}\left\{a\left(x_{1}, \cdots, x_{m}\right)\right\}=\left\{-x_{k} a\left(x_{1}, \cdots, x_{m}\right)\right\}$.

As in one variable case, we can easily verify the following:

Lemma 13. For each integer $k(1 \leq k$ $\leq m)$ we have

(1) If $\alpha$ is free of $x_{k}$, then $D_{k} \alpha=0$,

(2) $D_{k}(a+b)=D_{k} a+D_{k} b$,
(3) $D_{k}(a b)=\left(D_{k} a\right) b+a D_{k} b$,

(4) $D_{k}\left(\frac{b}{a}\right)=\frac{a D_{k} b-b D_{k} a}{a^{2}}$,

(5) $D_{j} s_{j}=1$ and $D_{j} s_{k}=0(j \neq k)$.

Remark. Lemma 13 shows that $\mathbf{C}\left[s_{1}, \ldots\right.$, $\left.s_{m}, D_{1}, \ldots, D_{m}\right]$ is the Weyl algebra.

Proposition 14.

$$
\begin{aligned}
& \frac{j_{1} ! \cdots j_{m} !}{\left(s_{1}-\alpha_{1}\right)^{j_{1}+1} \cdots\left(s_{m}-\alpha_{m}\right)^{j_{m}+1}} \\
& =\left\{x_{1}^{j_{1}} \cdots x_{m}^{j_{m}} e^{\alpha_{1} x_{1}+\cdots+\alpha_{m} x_{m}}\right\}
\end{aligned}
$$

Proof. Operating $D_{1}^{j_{1}} \cdots D_{m}^{j_{m}}$ to both sides of the equation in Proposition 12, we have the equation.

Lemma 15. Let $h_{1}(x, y)=\max (y-x, 0)$.

Then we have

$s_{y}^{2}\left\{e^{-c x} h_{1}(\alpha x, y)\right\}\{f(x, y)\}$

$$
=\left\{e^{-c x} \int_{\max \left(x-\frac{u}{\alpha}, 0\right)}^{x} e^{c u} f(u, y-\alpha x+\alpha u) d u\right\} .
$$

Proof. First we assume $y \geq \alpha x$. Then we have

$$
\begin{aligned}
& s_{y}^{2}\left\{e^{-c x} h_{1}(\alpha x, y)\right\}\{f(x, y)\} \\
& =s_{y}^{2}\{f(x, y)\}\left\{e^{-c x} h_{1}(\alpha x, y)\right\} \\
& =s_{y}^{2}\left\{\int_{0}^{x} e^{-c u} d u \int_{0}^{y} f(x-u, y-v) h_{1}(\alpha u, v) d v\right\} \\
& =s_{y}^{2}\left\{\int_{0}^{x} e^{-c u} d u \int_{\alpha u}^{y}(v-\alpha u) f(x-u, y-v) d v\right\}, \\
& =s_{y}^{2}\left\{\int_{0}^{x} e^{-c u} d u \int_{0}^{y-\alpha u}(y-\alpha u-w) f(x-u, w) d w\right\} \\
& =s_{y}\left\{\int_{0}^{x} e^{-c u} d u \int_{0}^{y-\alpha u} \frac{\partial(y-\alpha u-w)}{\partial y} f(x-u, w) d w\right\} \\
& =s_{y}\left\{\int_{0}^{x} e^{-c u} d u \int_{0}^{y-\alpha u} f(x-u, w) d w\right\} \\
& =\left\{\int_{0}^{x} e^{-c u} f(x-u, y-\alpha u) d u\right\},(x-u=t) \\
& =\left\{e^{-c x} \int_{0}^{x} e^{c t} f(t, y-\alpha x+\alpha t) d t\right\} .
\end{aligned}
$$

Now we consider the case $0<y<\alpha x$. Then we have

$$
\begin{aligned}
& s_{y}^{2}\left\{e^{-c x} h_{1}(\alpha x, y)\right\}\{f(x, y)\} \\
& =s_{y}^{2}\left\{\int_{0}^{x} e^{-c u} d u \int_{0}^{y} h_{1}(\alpha u, v) f(x-u, y-v) d v\right\} \\
& =s_{y}^{2}\left\{\int_{0}^{\frac{y}{\alpha}} e^{-c u} d u \int_{\alpha u}^{y}(v-\alpha u) f(x-u, y-v) d v\right\}, \\
& (w=y-v)
\end{aligned}
$$

$=s_{y}^{2}\left\{\int_{0}^{\frac{y}{\alpha}} e^{-c u} d u \int_{0}^{y-\alpha u}(y-\alpha u-w) f(x-u, w) d w\right\}$

$=s_{y}\left\{\int_{0}^{\frac{y}{\alpha}} e^{-c u} d u \int_{0}^{y-\alpha u} \frac{\partial(y-\alpha u-w)}{\partial y} f(x-u, w) d w\right\}$ 


$$
\begin{aligned}
& =s_{y}\left\{\int_{0}^{\frac{y}{\alpha}} e^{-c u} d u \int_{0}^{y-\alpha u} f(x-u, w) d w\right\} \\
& =\left\{\int_{0}^{\frac{y}{\alpha}} e^{-c u} f(x-u, y-\alpha u) d u\right\},(x-u=t) \\
& =\left\{e^{-c x} \int_{x-\frac{y}{\alpha}}^{x} e^{c t} f(t, y-\alpha x+\alpha t) d t\right\} .
\end{aligned}
$$

Theorem 16. Let $f$ be in $C$. Then we have

$$
\begin{aligned}
& \frac{1}{s_{x}+\alpha s_{y}+c}\{f(x, y)\} \\
& =\left\{e^{-c x} \int_{\max \left(x-\frac{y}{\alpha}, 0\right)}^{x} e^{c u} f(u, y-\alpha x+\alpha u) d u\right\} .
\end{aligned}
$$

Proof. Let $w(x, y)$ be the right side of the equation. Then it is clear that $w(x, y)$ vanishes both on $x$-axis and on $y$-axis. Hence $s_{x}=d_{x}$ and $s_{y}=d_{y}$. Routine calculation shows $\left(d_{x}+\alpha d_{y}\right.$ $+c) w(x, y)=f(x, y)$. Therefore $w(x, y)=$ $\frac{1}{s_{x}+\alpha s_{y}+c} f(x, y)$.

Corollary. $\frac{1}{s_{x}+\alpha s_{y}+c}=s_{y}^{2}\left\{e^{-c x} h_{1}(\alpha x, y)\right\}$.

Proof. This follows from Lemma 15, Theorem 16 and Proposition 2.

\section{Applications.}

Example 1. Find the solution of the equation

$\left(d_{x}+\alpha d_{y}+c\right)\{w(x, y)\}=\{g(x, y)\} \quad(\alpha>0)$. with $w(0, y), w(x, 0)$ and $g(x, y)$ given.

Setting $z(x, y)=w(x, y)-w(0,0)$ and $f(x, y)=g(x, y)-c w(0,0)$, the equation is equivalent to

$$
\begin{gathered}
\left(d_{x}+\alpha d_{y}+c\right)\{z(x, y)\}=\{f(x, y)\} \quad(\alpha>0), \\
\text { with } z(0,0)=0 .
\end{gathered}
$$

Since $d_{x}=s_{x}-s_{x} r_{x}$ and $d_{y}=s_{y}-s_{y} r_{y}$, we can rewrite the equation as

$$
\begin{aligned}
z= & \frac{s_{x}}{s_{x}+\alpha s_{y}+c}\{z(0, y)\} \\
& +\frac{\alpha s_{y}}{s_{x}+\alpha s_{y}+c}\{z(x, 0)\} \\
& +\frac{1}{s_{x}+\alpha s_{y}+c}\{f(x, y)\} .
\end{aligned}
$$

The first and the second terms of the right hand side are determined uniquely by the values of $z$ on $\{0\} \times[0, \infty)$ and on $[0, \infty) \times\{0\}$ respectively. The third term is the particular integral which vanishes both on $\{0\} \times[0, \infty)$ and on $[0$, $\infty) \times\{0\}$. The above equation shows that any solution $z$ with $z(x, 0)=0$ and $z(0, y)=0$ is uniquely determined and is equal to the third term. We can express each term in more familiar forms: If $y>\alpha x$, then

$$
\begin{gathered}
\frac{s_{x}}{s_{x}+\alpha s_{y}+c} z(0, y) \\
=s_{x}\left\{e^{-c x} \int_{0}^{x} e^{c u} z(0, y-\alpha x+\alpha u) d u\right\} \\
=s_{x}\left\{\int_{0}^{x} e^{-c v} z(0, y-\alpha v) d v\right\},(x-u=v) \\
=e^{-c x} z(0, y-\alpha x) .
\end{gathered}
$$

If $y \leq \alpha x$, then

$$
\begin{aligned}
& \frac{s_{x}}{s_{x}+\alpha s_{y}+c} z(0, y) \\
& =s_{x}\left\{e^{-c x} \int_{x-\frac{y}{\alpha}}^{x} e^{c u} z(0, y-\alpha x+\alpha u) d u\right\} \\
& =s_{x}\left\{\int_{0}^{\frac{y}{\alpha}} e^{-c v} z(0, y-\alpha v) d v\right\},(x-u=v) \\
& =d_{x}\left\{\int_{0}^{\frac{y}{\alpha}} e^{-c v} z(0, y-\alpha v) d v\right\} \\
& =0 .
\end{aligned}
$$

Therefore we have

$$
\begin{aligned}
& \frac{s_{x}}{s_{x}+\alpha s_{y}+c} z(0, y) \\
& = \begin{cases}e^{-c x} z(0, y-\alpha x), & (y>\alpha x) \\
0, & (y \leq \alpha x) .\end{cases}
\end{aligned}
$$

We have a similar formula for the second term. To make the solution continuous on the boundary $y=\alpha x$, we impose the condition $z(0,0)=0$. Therefore, by Theorem 16, we have $z(x, y)$

$$
=\left\{\begin{array}{l}
\left\{e^{-c x} z(0, y-\alpha x)\right\}+ \\
\left\{e^{-c x} \int_{0}^{x} e^{c u} f(u, y-\alpha x+\alpha u) d u\right\},(y>\alpha x), \\
\left\{e^{\frac{-c y}{\alpha}} z\left(x-\frac{y}{\alpha}, 0\right)\right\}+ \\
\left\{\frac{1}{\alpha} e^{\frac{-c y}{\alpha}} \int_{0}^{y} e^{\frac{c v}{\alpha}} f\left(x-\frac{y}{\alpha}+\frac{v}{\alpha}, v\right) d v\right\},(0 \leq y \leq \alpha x) .
\end{array}\right.
$$

Example 2. Let $f(x, y)$ be in $C$. Then find the solution of

$$
\left(d_{x}-\alpha d_{y}+c\right)\{z(x, y)\}=\{f(x, y)\}
$$

with $z(0, y)=g(y)$ and $\alpha>0$. The equation is equivalent to

$$
\left(s_{x}-\alpha d_{y}+c\right)\{z(x, y)\}=s_{x}\{g(y)\}+\{f(x, y)\} .
$$
Hence

$$
\begin{aligned}
& z(x, y)=s_{x} \frac{1}{s_{x}-\alpha d_{y}+c}\{g(y)\} \\
& \quad+\frac{1}{s_{x}-\alpha d_{y}+c}\{f(x, y)\} \\
& =\frac{\partial}{\partial x} \int_{0}^{x} e^{-c u} g(y+\alpha u) d u \\
& \quad+\int_{0}^{x} e^{c(u-x)} f(u, y+\alpha x-\alpha u) d u
\end{aligned}
$$


$=e^{-c x} g(y+\alpha x)+\int_{0}^{x} e^{c(u-x)} f(u, y+\alpha x-\alpha u) d u$

This gives the unique solution.

Example 3. Find the solution of $\left(d_{x}^{2}-\alpha^{2} d_{y}^{2}\right)\{z(x, y)\}=\{f(x, y)\}$, with $z(0,0)=0$. Assume that boundary conditions $z(0, y)$ and $z_{x}(0, y)$ are given. Then, in the region $y \geq \alpha x$, the solution is uniquely determined as follows. Applying the formula in Example 2, we have $\left(d_{x}+\alpha d_{y}\right)\{z(x, y)\}$

$$
\begin{aligned}
=\left\{z_{x}(0, y+\alpha x)\right. & +\alpha z_{y}(0, y+\alpha x) \\
& \left.+\int_{0}^{x} f(u, y+\alpha x-\alpha u) d u\right\} .
\end{aligned}
$$

Now by the formula in Example 1, we have

$z(x, y)=z(0, y-\alpha x)$

$+\int_{0}^{x} z_{x}(0,(y-\alpha x+\alpha u)+\alpha u) d u$

$+\alpha \int_{0}^{x} z_{y}(0,(y-\alpha x+\alpha u)+\alpha u) d u$

$+\int_{0}^{x} d v \int_{0}^{v} f(u,(y-\alpha x+\alpha v)+\alpha v-\alpha u) d u$.

Here we have $\alpha \int_{0}^{x} z_{y}(0, y-\alpha x+2 \alpha u) d u=$

$$
(z(0, y+\alpha x)-z(0, y-a x)) / 2 .
$$

Therefore we have finally

$$
\begin{aligned}
& z(x, y)=\frac{1}{2} z(0, y-\alpha x)+\frac{1}{2} z(0, y+\alpha x) \\
& +\int_{0}^{x} z_{x}(0, y-\alpha x+2 \alpha u) d u \\
& +\int_{0}^{x} d v \int_{0}^{v} f(u, y-\alpha x+2 \alpha v-\alpha u) d u .
\end{aligned}
$$

In the region $y \leq \alpha x$, we have also the solution exchanging the role of $x$ and $y$.

\section{References}

[1] J. Mikusiński and T. K. Boehme: Operational Calculus. Pergamon (1987).

[2] K. Yosida and S. Okamoto: A note on Mikusiński's operational calculus. Proc. Japan Acad., 56A, $1-3$ (1980).

[3] K. Yosida: Functional Analysis. Springer (1966). 